\title{
Summary Report:
}

\section{Nanomedicines: Technical and Regulatory Perspectives Co-sponsored by: USP, AAPS, and FIP}

Summarized on behalf of the FIP Dissolution/In Vitro Performance Focus Group in Collaboration with USP by Amy R. Barker, Margareth Marques*, Johannes Krämer, Vinod P. Shah, and Horst-Dieter Friedel

e-mail: mrm@usp.org

\section{INTRODUCTION}

$f$ you were one of the nearly 150 people that attended the Nanomedicines workshop in Rockville, Maryland, on March 20-22th, 2017, you were able to confirm that medicines containing nanomaterials continue to be an evolving space in academia, industry, and regulatory environments. During the 3-day workshop, 30 speakers presented their experience and expertise covering the areas of new product development, critical quality attributes, analytical tools, and regulatory perspectives. Many pharmaceutical formulations are complex in order to treat critical medical indications for patients. There are studies underway to identify and improve paths to deliver critical medicines directly to targeted cells for longer periods of time. Nanomaterials research aims to reproducibly manufacture medicines and optimize treatment for multiple disease states. Although there are already many accomplishments in the area of medicines containing nanomaterials, there is clearly much to learn and accomplish in the future.

Nanoparticles are not uniformly defined across environments (global regulatory, food, agriculture). Nanoscale is an arbitrary classification that ranges from 1-1000 nm. Products may be defined as nanomaterials based on size, shape, or surface reactivity, and the definition is sometimes related to product purpose or function as much as size. Multiple cross-functional teams exist today to develop guidelines that align a worldwide science-based regulatory view. Some examples of groups and committees with new or existing guidance include CDER nanotechnology guidance, USP joint subcommittee on nanotechnology, ASTM E56 committee on nanotechnology, ISO TC 220 nanotechnologies, EMA reflection paper on nanomedicines, and the US FDA nanotechnology task force subcommittee on standards.

\section{DEVELOPMENT}

Nanoparticles are an area of increasing development to modify clinical applications of novel active ingredients or drug products to increase the therapeutic index using targeted therapy and/or to increase half-life. Nanotechnologies may be used to increase bioavailability, change biodistribution, increase drug action, stabilize material, or target delivery.

There are many types of nanoparticles with new technologies and development products ranging across multiple sizes including nano and micro particulates. Some areas of research also include non-biological complex drugs (NBCDs), which may follow small molecule delivery routes, biosimilar delivery routes, or a hybrid of the two. Other areas of medicines containing nanomaterials research include PEGylated compounds (covalent or noncovalent attachment of polyethylene glycol), liposomes, antibody drug conjugates, polymer drug conjugates, polymer micelles, and more. PYRROLIDONylation reagents are an alternative to PEGylation.

In each area of medicines containing nanomaterials and nanotechnology research, some of the most complex aspects include in vivo interaction. Measurements of biologic activity, toxicity, and clearance are key aspects to early product development. Common complexities when developing models of cellular uptake, simulation of intracellular trafficking and site of drug release, and simulation of efficiency of different drug delivery systems to release drug intracellularly occur with nanomedicines. Toxicity can differ depending on the environment (i.e., toxicity in lysosomal fluid may be higher due to lack of agglomeration versus water where the coating may be intact).

The European Union Nanomedicine Characterisation Laboratory (EU-NCL) provides perspectives on start-up concepts for nanomedicines. The organization provides knowledge, expertise, and assistance with development of the critical data packages as well as generating data to support submission of new products used in cancer therapy. They provide a free-of-charge consortium of 
laboratories with a project manager as well as a number of scientists with a variety of expertise. EU-NCL offers prescreening, in vitro and cytotoxicity, in vivo evaluations, case-by-case questions, as well as partnering with regulators. The organization also has a survey that they performed with 18 regulators that was published during 2017. In their presentation, EU-NCL noted that their goal is to help streamline nanomedicine evaluations and provide development support to the product developer.

Although development of new nanomedicines is complex, there are a couple of products on the market that may be used as examples for issues such as manufacturing scale-up, submission discussions, initial ideas for critical process parameters, as well as identification of critical quality attributes. Because newer products will be more complex and scientific and regulatory knowledge have evolved since approval of the first drug product containing nanomaterials, consideration must be taken to include recent learning in all aspects of development of novel medicines.

\section{Critical Quality Attributes (CQA) and Critical Process Parameters (CPP)}

Identification of CQAs evolves as one of the most important aspects of evaluation of nanotechnologies. As drug delivery does not follow the more traditional paths and the medicine is frequently a novel formulation, there is not an established template for identifying CQAs either as manufacturing process parameters or as analytical measurements to assure quality of the product. Each novel product will require holistic technical understanding to identify both CPPs and CQAs.

To identify CQAs, it is necessary to characterize the product. The quality-by-design approach should be employed when developing a process to control manufacturing. ICH Q8 and Q9 are the relevant guidelines for identification of CQAs and CPPs. CQAs that are unique to a nanoparticle include understanding polymers or other raw materials, the drug load, counter ion content, particle size distribution, and the product morphology. Some of the most frequent properties evaluated as part of manufacturing of nanomedicines include particle size, shape, surface chemistry, surface charge, flow characteristic, rigidity, binding strength, and elemental composition.

Definition of CQAs will remain a topic of interest, as the definition is complex and may sometimes be seemingly subjective. Typical properties evaluated, characterization tools, and control will differ for products. Discrepancies may occur across products or technologies for identification of one attribute or another as critical, requiring in-depth understanding of analytical techniques, process characteristics, and scale-up complexities.

\section{Analytical Tools}

Analytical tools are leveraged to generate predictive modeling determinations during development. Techniques for predictive in vivo information include those that measure surface chemistry at nano, micro, and macro scales for both inorganic and organic particles. As excretion levels are driven by size, among other measurements, this becomes one of the more critical parameters for modeling. Typical excretion patterns include less than $6 \mathrm{~nm}$ through kidney, 6-8 nm through lymph nodes, and greater than $8 \mathrm{~nm}$ through spleen or liver. Electrophoresis is a strong option for a technology to measure dendrimer quality (size, shape, purity). Techniques and models are also necessary to demonstrate understanding of biodistribution including in vivo clearance, localized accumulation, release of payload, delivery of efficacious dose, and dose-dependent toxicity.

Required measurements that demonstrate product performance typically include and are not limited to API in vitro release, particle size distribution, and surface chemistry characteristics. Each of these measurements are most frequently identified and defined early in development. They are also then necessary to track through manufacturing in early, clinical, and commercial phases for a product. While the measurement technique may evolve from a development mode measurement to a QC measurement, it is necessary to demonstrate the relationship across the measurements and methodologies.

Methods that most frequently emerge within the $\mathrm{QC}$ environment for drug products containing nanomaterials include high-order structure, glycosylation, amino acid analysis, charged variants, surface morphology, and density. Particle size distribution (PSD) is typically measured by dynamic light scattering (DLS), but transmission electron microscopy (TEM) can show if differences in morphology exist. Charge may also impact accumulation. Orthogonal approaches are important to confirm both size and morphology. Other methods to characterize nanoparticle systems are size-exclusion chromatography coupled with UV, RI, and LS detectors (SEC-MALS), or nanoparticle tracking analysis (NTA). The most frequently used techniques in submissions (FDA) are DLS, laser diffraction, and microscopic/imaging techniques. For a broad size distribution, it is important to control the whole PSD rather than the mean size only. For setting specifications for the PSD, the drug product 
properties, manufacturing process, and particle sizing techniques have to be considered. Typically, D10, D50, or D90 are specified for the PSD. Validation of a particle sizing method differs from validation of other methods described in ICH Q2 because specificity, range, detection limit, quantification limit, accuracy, and linearity are not required. Data for precision (repeatability, intermediate precision, reproducibility) and robustness should be generated for validation of the particle sizing method.

Chemical properties are frequently measured using differential scanning calorimetry (DSC), thermal gravimetric analysis (TGA), energy dispersive $\mathrm{X}$-ray spectroscopy, and inductively coupled plasma mass spectrometry (ICP/ MS). Challenges in QC measurements arise due to lack of robustness within a particular technique as well as differences across vendors for given instrumentation, technologies, and measurements. Finally, techniques required to characterize samples are more complex and most are destructive in nature.

Some examples of analytical properties that are common for nanoparticles include chemical composition, solubility/ suspendability, thermal analysis, redox capacity, zeta potential as a measure of surface charge, and $\mathrm{pH} /$ isoelectric point. Depending on the CQAs, all or some of these property determinations will be combined with other measurements to demonstrate product quality as part of batch release.

Another important method that applies to both development and QC testing for medicines containing nanomaterials is in vitro release. Development of a relevant in vitro release method is challenging due to complications of modeling in vivo release from the matrix. In addition, definition of a robust in vitro release technology frequently involves application of non-traditional dissolution techniques. The in vitro release method must be capable of releasing the drug from the nanomaterial matrix, separating dissolved from undissolved particles, preparing a solution that will be stable from the time of sample collection to the time of analysis, detecting, analyzing, and quantitating the material of interest. Different release measurement methods will be required for different types of formulations. Depending on the phase of development, the samples could be complex (blood or serum) or more simple. For complex matrices such as liquid dispersions, dissolution may require sample pre-preparation or application of dialysis with an appropriate diffusion membrane. Stir rate and temperature impact transport of material across the membrane, and it is critical that a membrane remain intact throughout the experiment.
Detection methods may require sample derivatization or technologies such as mass spectrometry.

Since release from nanoparticles is likely to be more time consuming, automation is recommended for consideration to create a closed system and avoid evaporation. For slower release, multiple measurements must be considered as part of the release profile. Fiber optics may be employed to assist with the higher number of measurements. If detection probes are used and the matrix is complex, treatment of the probes may be necessary to avoid accumulation of sample at the detector. Probe placement in the in vitro release vessel must also be evaluated to ensure placement in the most robust location and to demonstrate lack of interference with the release process and measurement. Due to slow release and the necessity for high method/detector sensitivity, it is possible that integral calculations such as first or second derivatives will be required to differentiate signal from noise. The $\mathrm{QC}$ method must provide information as to the consistency of the batch as well as batch-to-batch similarity. If the method is capable of in vivo correlation, it will also provide information about differences between batches.

Evaluation of drug products containing nanomaterials is a newer concept in the commercial space, and many of the technologies and techniques required to evaluate medicinal quality do not yet maintain general compendial chapters. International working groups are being established to address these needs, and it is expected that additional compendial general chapters will be established in the coming years.

\section{Regulatory Perspectives}

Throughout the workshop, it was noted that nomenclature for medicines containing nanomaterials is inconsistent throughout regulatory as well as industry. While "nano" is approximately 1-100 nm, it sometimes also includes 1000 to $10,000 \mathrm{~nm}(1-10 \mu \mathrm{m})$. There are many committees that have been established globally to work on terminology as well as nanomedicine concepts. The US FDA has a nanotechnology task force as well as a nanotechnology working group that puts out guidance for industry and works on cross-functional external teams. A CDER nanotechnology guidance is being drafted, there is a USP joint sub-committee on nanotechnology, and an ASTM E56 committee on nanotechnology has been established; there is an ISO TC 220 nanotechnologies team, and there are many EMA expert groups on nanomedicines that exist and have drafted guidance, established collaborations with the International Pharmaceutical Regulatory Forum 
(IPRF), and have finalized publications. These are just a few of the working groups and committees that were discussed during the workshop.

An EU regulation on drug products containing nanomaterials was summarized to provide a risk-based assessment as well as classification information for nanomedicines. Classification of devices containing nanomaterials is based on particle release, PSD and persistence, hazard assessment, and risk characterization assessment. In addition, the EMA has established Priority Medicines (PRIME), which aims to strengthen support to medicines that target an unmet medical need. The PRIME scheme encourages early work with the agency to engage in dialogue, working collaboratively on a scientific advice team, and obtaining a coordinated European position.

Many issues may impact regulatory reviews for nanotechnology-based products due to their diversity and complex structures. It was recommended by the represented agencies to initiate discussions early with regulatory bodies to assure complete understanding of the product as well as critical attributes. Review issues such as pharmacokinetic profiles, product performance and safety, side effects, specialized analytical testing, challenges in physical and chemical stability, and control of manufacturing are a few items that may be a focus during submission planning and early regulatory discussions. Product development should follow ICH Q8 (QTPP (quality target product profile), CQAs, process risk assessment, design space, control strategy, continual improvement), Q9, and Q10. While there may be multiple relevant attributes, the critical ones will be dependent on the product.

\section{CONCLUSIONS}

Although many accomplishments have been recognized in the space of medicines containing nanomaterials, there is much work ongoing and there is more room to learn. In the future, clarification in the global definition of nanoparticles will help to define regulations for new products. In addition, consistency across global practices, sharing and publication of learning as well as guidance will provide critical information to product developers. As new technologies are employed for analysis of CQAs, compendial chapters to harmonize expectations will assist in implementation of methods that are ready for regulatory evaluation. Finally, early discussions with regulatory agencies are necessary to collaborate and develop submissions to support safe and efficacious products for novel therapies.

\section{SPEAKER SUMMARY FOR USP WORKSHOP PROGRAM}

Nanomedicines: Technical and Regulatory Perspectives Co-sponsored by: USP, AAPS, and FIP March 22-24, 2017

\begin{tabular}{|c|c|}
\hline Presentation Title & Speaker \\
\hline $\begin{array}{l}\text { Nanotechnology - Common Language for Nomenclature, Standards (ISO, ASTM) and } \\
\text { Data Reporting }\end{array}$ & Fred Klaessig, Pennsylvania Bio Nano systems, LLC \\
\hline Non-Biological Complex Drugs: Regulatory Challenges & Daan Crommelin, Ultrecht University \\
\hline $\begin{array}{l}\text { Quality Considerations and Regulatory Perspectives for Drug Products Containing } \\
\text { Nanomaterials: FDA Perspective }\end{array}$ & Katherine Tyner, US Food and Drug Administration \\
\hline $\begin{array}{l}\text { Quality Considerations and Regulatory Perspectives for Drug Products Containing } \\
\text { Nanomaterials: European Perspective }\end{array}$ & $\begin{array}{l}\text { Rene Thuermer, Federal Institute for Drugs and Medical Devices, } \\
\text { Germany }\end{array}$ \\
\hline Perspectives on the Start-up of EU-NCL & Susanne Bremer-Hoffmann, European Commission \\
\hline USP Perspectives for Drug Products Containing Nanomaterials & Anthony Hickey, Research Triangle Institute \\
\hline $\begin{array}{l}\text { Immunological characterization of nanotechnology-based formulations: Challenges } \\
\text { and considerations }\end{array}$ & Marina Dobrovolskaia, Leidos Biomedical Research Inc. \\
\hline $\begin{array}{l}\text { Use of Mononuclear Phagocyte Platforms to Characterize Nanomaterials, } \\
\text { Nanoparticles and Colloids }\end{array}$ & $\begin{array}{l}\text { William C. Zamboni, Eshelman School of Pharmacy, University of } \\
\text { North Carolina at Chapel Hill }\end{array}$ \\
\hline Challenges in the Release Testing of Next Generation Nanomedicines & $\begin{array}{l}\text { Matthias Wacker, Fraunhofer-Institute for Molecular Biology and } \\
\text { Applied Ecology, Germany }\end{array}$ \\
\hline Development of In Vitro Release Procedure for Complex Nanotechnology Products & Ye Zhang, US Food and Drug Administration \\
\hline Development of In Vitro Release Procedure for Small Molecules and Biologics & Ajit Narang, Genetech, Inc. \\
\hline Analytical Aspects & $\begin{array}{l}\text { Scott McNeil, Nanotechnology Characterization Laboratory, } \\
\text { National Cancer Institute/NIH }\end{array}$ \\
\hline Current and Emerging Techniques & Tao Lu Lowe, University of Tennessee Health Science Center \\
\hline
\end{tabular}




\begin{tabular}{|c|c|}
\hline Industrial Perspective on Nanomedicine characterization Strategies & Don Parsons, Moderna Therapeutics \\
\hline Characterization of Nanomaterials - Best Practices and Databases & $\begin{array}{l}\text { Martin Fritts, National Institute of Standards and Technology/ } \\
\mathrm{NCl}\end{array}$ \\
\hline Characterization of Nanomaterials & Christie Sayes, Baylor University \\
\hline $\begin{array}{l}\text { Complementary Technologies for Characterization of Particles in the Nano/Colloidal } \\
\text { Range }\end{array}$ & Aaron Krueger, KBI Biopharma, Inc. \\
\hline The Use of Zeta Potential for the Characterization of Nanomaterials & Alan Rawle, Malvern Instruments \\
\hline In Situ Spectroscopy with Nanoparticles & Alexis Guillot, PHAST GmbH \\
\hline Characterization of Liposomes & Daan Crommelin, Utrecht University \\
\hline $\begin{array}{l}\text { Oligonucleotide Lipid Nanoparticles: Development, Process Optimization and } \\
\text { Specification Setting }\end{array}$ & Andrew Latham, Merck Research Laboratories \\
\hline $\begin{array}{l}\text { The Importance of Multi-Dimensional Characterization in the Pharmaceutical } \\
\text { Development and Control of siRNA Nanoparticle Drug Product }\end{array}$ & Jingtao Zhang, Merck \\
\hline Biocompatible Nanoparticles for Targeted Delivery & Sylvia Wagner, Fraunhofer Institute for Biomedical Engineering \\
\hline $\begin{array}{l}\text { Biomaterials for Polymeric Particulate Delivery Systems: Technical and Regulatory } \\
\text { Considerations }\end{array}$ & Sudhir S. Chakavarthi, Bristol-Myers Squibb \\
\hline Particle Size and Shape Characterization: Current and Emerging Techniques & Mario Hubert, Bristol-Myers Squibb \\
\hline Impact of Drug Nanocrystal Aggregation in Oral Dosage Forms & Ecevit Bilgili, New Jersey Institute of Technology \\
\hline End of life for Nanomedicines & Christie Sayes, Baylor University \\
\hline Scientific and Regulatory Considerations on Particle Size Analysis of Nanomaterials & Zhigang Sun, US Food and Drug Administration \\
\hline
\end{tabular}

